\title{
Molecular Docking Study of Aspirin and Aspirin Derivatives
}

\author{
Ramjith.U.S ${ }^{1 *}$, Ayda Cherian ${ }^{2}$, Navneeth Krishna Manoj ${ }^{1}$, Sameer P.A ${ }^{1}$, \\ Smrithi Radhakrishnan ${ }^{1}$ \\ ${ }^{I}$ Department of Pharmaceutical Chemistry, Crescent College of Pharmaceutical Sciences, Madayipara, \\ Payangadi (R.S), Kannur, Kerala -670358. \\ ${ }^{2}$ Department of Pharmaceutical Chemistry, The Dale View College of Pharmacy and Research Centre, \\ Punalal, Poovachal, Thiruvanathapuram-695575.
}

\begin{abstract}
The molecular docking studies were performed on aspirin and aspirin derivatives using HVR protein. The compounds SR-03, SR-02, SR-04 were found to show best docking scores towards HVR protein (HIV protease receptor) indicating that these compounds may be screened for in vivo anti-HIV activity. It is concluded that electron withdrawing groups like $(\mathrm{R}=-\mathrm{OH}$ substituted aryl) group when attached to carboxylic group of aspirin increases the in vitro anti-oxidant activity and affinity for HIV protein while electron releasing groups like $\left(\mathrm{R}=-\mathrm{CH}_{3},-\mathrm{C}_{2} \mathrm{H}_{5},-\mathrm{CH}\left(\mathrm{CH}_{3}\right)_{2},-\mathrm{CH}_{2}-\mathrm{CH}_{2}-\mathrm{CH}_{2}-\mathrm{CH}_{3}\right)$ decreases affinity for HIV protein. Further studies are required to establish their exact mechanism of action.
\end{abstract}

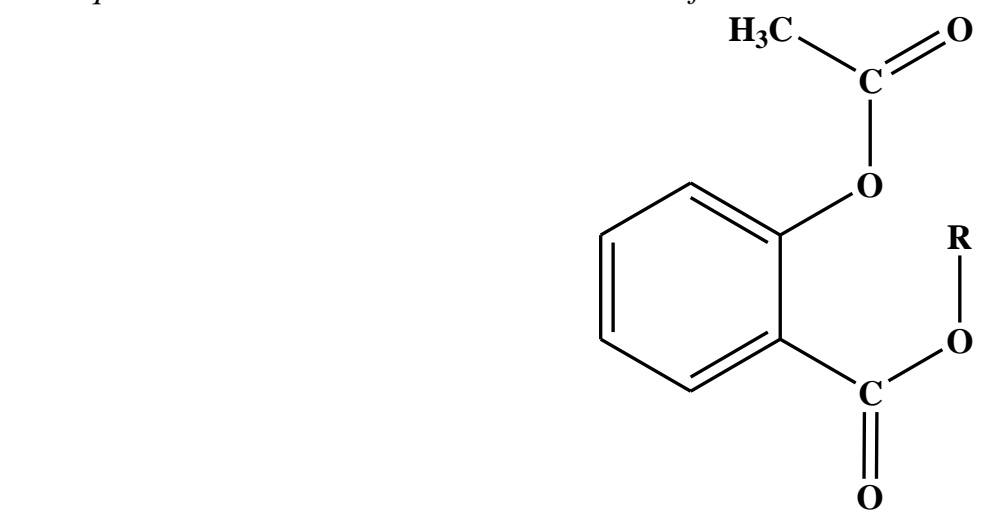

$\mathrm{R}=\mathrm{CH}_{3}, \mathrm{C}_{2} \mathrm{H}_{5}, \mathrm{C}_{3} \mathrm{H}_{7}, \mathrm{C}_{4} \mathrm{H}_{9}, \mathrm{C}_{6} \mathrm{H}_{5}, \mathrm{C}_{6} \mathrm{H}_{5} \mathrm{CH}_{2}, \mathrm{C}_{6} \mathrm{H}_{5} \mathrm{O}$.

Keywords: Aspirin, HVR protein, anti-HIV activity.

\section{Introduction}

Molecular docking is a method to predict the preferred orientation of one molecule to a second when bound to each other to form a stable complex. Computers and programs (software's) are used to predict or simulate the possible reaction (and interactions) between two molecules based on their three dimensional structures. The need for a rapid search for small molecules that may bind to targets of biological interest is of crucial importance in the drug discovery process. One way of achieving this is the in silico or virtual screening (VS) of large compound collections to identify a subset of compounds that contains relatively many hits against the target, compared to a random selection from the collection. The compounds that are virtually screened can be obtained from corporate or commercial compound collections, or from virtual compound libraries. If a threedimensional (3D) structure or model of the target is available, a commonly used technique is structure-based virtual screening (SBVS). Here a so-called 'docking program' is used to place computer-generated representations of a small molecule into a target structure (e.g., the active site of an enzyme) in a variety of positions, conformations and orientations.

Each such docking mode is called a 'pose'. In order to identify the energetically most favourable pose (also referred to as 'pose prediction'), each pose is evaluated ('scored') based on its complementarity to the target in terms of shape and properties such as electrostatics. A good score for a given molecule indicates that it is potentially a good binder. This process is repeated for all molecules in the collection, which are subsequently rank-ordered by their scores (i.e., their predicted affinities). This rank-ordered list is then used to select for purchase, synthesize or biologically investigate only those compounds that are predicted to be the most active. Assuming that both the poses and the associated affinity scores have been predicted with reasonable accuracy, this selection will contain a relatively large proportion of active molecules, i.e., it will be 'enriched' with actives compared to a random selection. 
POSING-The process of determining whether a given conformation and orientation of a ligand fits the active site. This is usually a fuzzy procedure that returns many alternative results.

The 2 stages of docking include:

- Pose generation- Place the ligand in the binding site.

- Pose selection- Determine the proper pose.

SCORING- The pose score is a measure of the fit of a ligand into the active site. Scoring during the posing phase usually involves simple energy calculations (electrostatic, van der Waals, ligand strain). Further rescoring might attempt to estimate more accurately the free energy of binding (delta $G$ and therefore $\mathrm{K}_{\mathrm{A}}$ ) perhaps including properties such as entropy and solvation.

\section{HOW DOCKING IS DONE-}

Typically, types of file or input are needed; one for the ligand/small molecule and another one for the target/receptor. The ligand or small molecule can be built using suitable software or taken from available databases. The protein or receptor's structure can either be downloaded from several databases, for example protein databank (it can be built based on a template using softwares with amino acids/biopolymer construction features (called homology modelling). The success of a program mainly depends on two components:

- The search algorithm

- The scoring function

\section{- The Search Algorithm-}

The search algorithm is a process where all possible conformations and orientations of the complex (the paired ligand and protein) in a space (the binding site of interest) are being searched. If the ligand is flexible, then the program will calculate the energy for each rotations made of each and every rotatable bonds it can find. The same goes for the protein/receptor. For each and every rotation of the side chain of the amino acids (in the binding site), the program will calculate the energy involved. Each energy value calculated will be presented as a "snapshot" of the pair.

In each of the snapshot, you will be able to see what kind of interactions are involved and which atoms are in contact or close proximity making any kinds of bonding such as hydrogen bonds, hydrophobic interactions and many more. Each of the "snapshots" of the pair or the complex is called the binding "pose".

Search Algorithms Used-

- Systematic Docking

-Brute Force

-Fragmentation

-Database

- Heuristic Docking

-Monte Carlo

-Genetic Algorithms

-Tabu Search

- Simulations Docking

-Molecular Dynamics

-Gradient (Energy) Methods

- The Scoring Function-

. Molecular mechanics force field (physics based calculations) is used to estimate the energy of each pose. Each and every pose will come with an energy value and the scoring function of the program will rank the poses accordingly (normally in a descending manner). The lower (negative) the energy of the pose, the more stable the complex will be, and more likely the possibility of the binding will happen.

The scoring function is a process where the program takes a binding pose and gives a number to indicate the likelihood whether the binding interaction is favourable or not.

$>$ Force Field based scoring function

- Energy of the interaction and internal energy of the ligand.

- Combination of: Van der Waals, Lennard Jones, electrostatic energy etc.

- E.g. D-Score, GoldScore, AutoDock, CHARMM.

$>$ Empirical scoring functions

- Trying to reproduce experimental observed docking behaviours by means of formulas.

- Usually the sum of uncorrelated terms.

- E.g. LUDI, F-Score, SCORE, X-SCORE.

$>$ Knowledge based scoring function

- Trying the deduce rules form experiments.

- E.g. Drug Score, PMF. 
Geometrical scoring function

- Based on shape complementarity.

- E.g. Connelly Surface, Soft Belt Scoring.

$>$ Consensus scoring function

- Hybrid versions

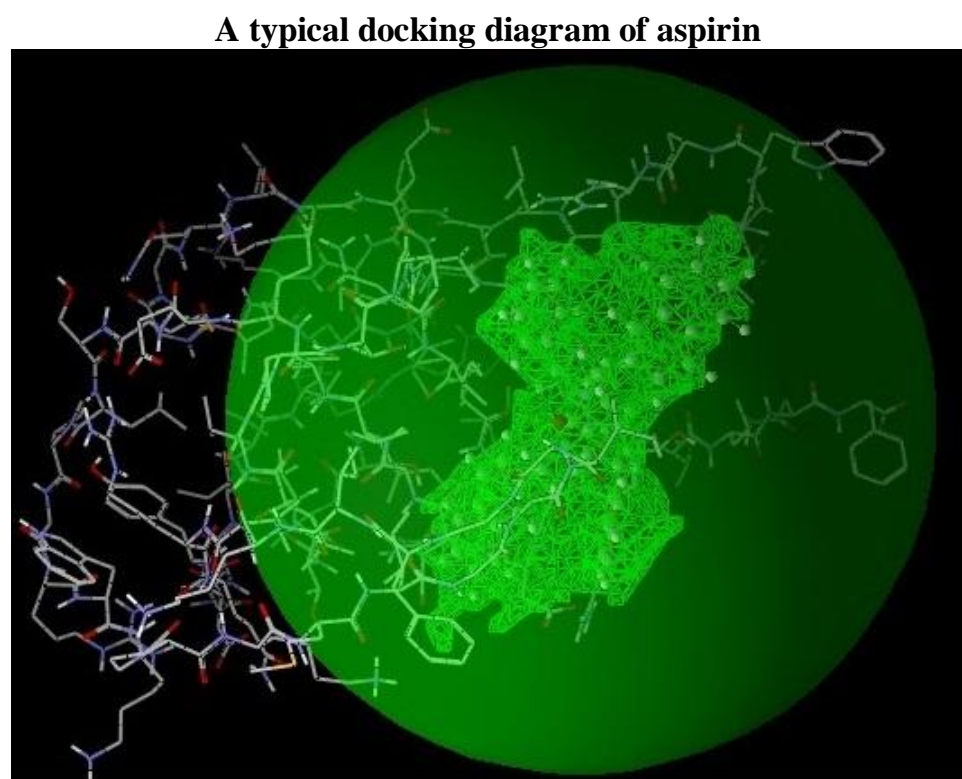

\section{APPLICATIONS}

Docking is most commonly used in the field of drug design-

Most drugs are small organic molecules, and docking may be applied to:

- Hit identification - Docking combined with a scoring function can be used to quickly screen large databases of potential drugs in silico to identify molecules that are likely to bind to protein target of interest.

- Lead optimization - Docking can be used to predict in where and in which relative orientation a ligand binds to a protein (also referred to as the binding mode or pose). This information may in turn be used to design more potent and selective analogues.

- Bioremediation - Protein ligand docking can also be used to predict pollutants that can be degraded by enzymes.

- Prediction of biological activity.

- Binding site identification.

- Enzymatic reactions mechanisms.

- Protein engineering.

- Protein-protein or protein-nucleic acid interactions.

- Structure function studies.

- De-orphaning of a receptor. ${ }^{[1,2,3,4,5,6]}$ 
Docking Study of Aspirin and Aspirin Derivatives

Protein used for docking: $1 \mathrm{HVR}$ (HIV-1 protease) ${ }^{7}$

Software used: MVD2010.4.0.

Table: 1- Compound Code and Structure

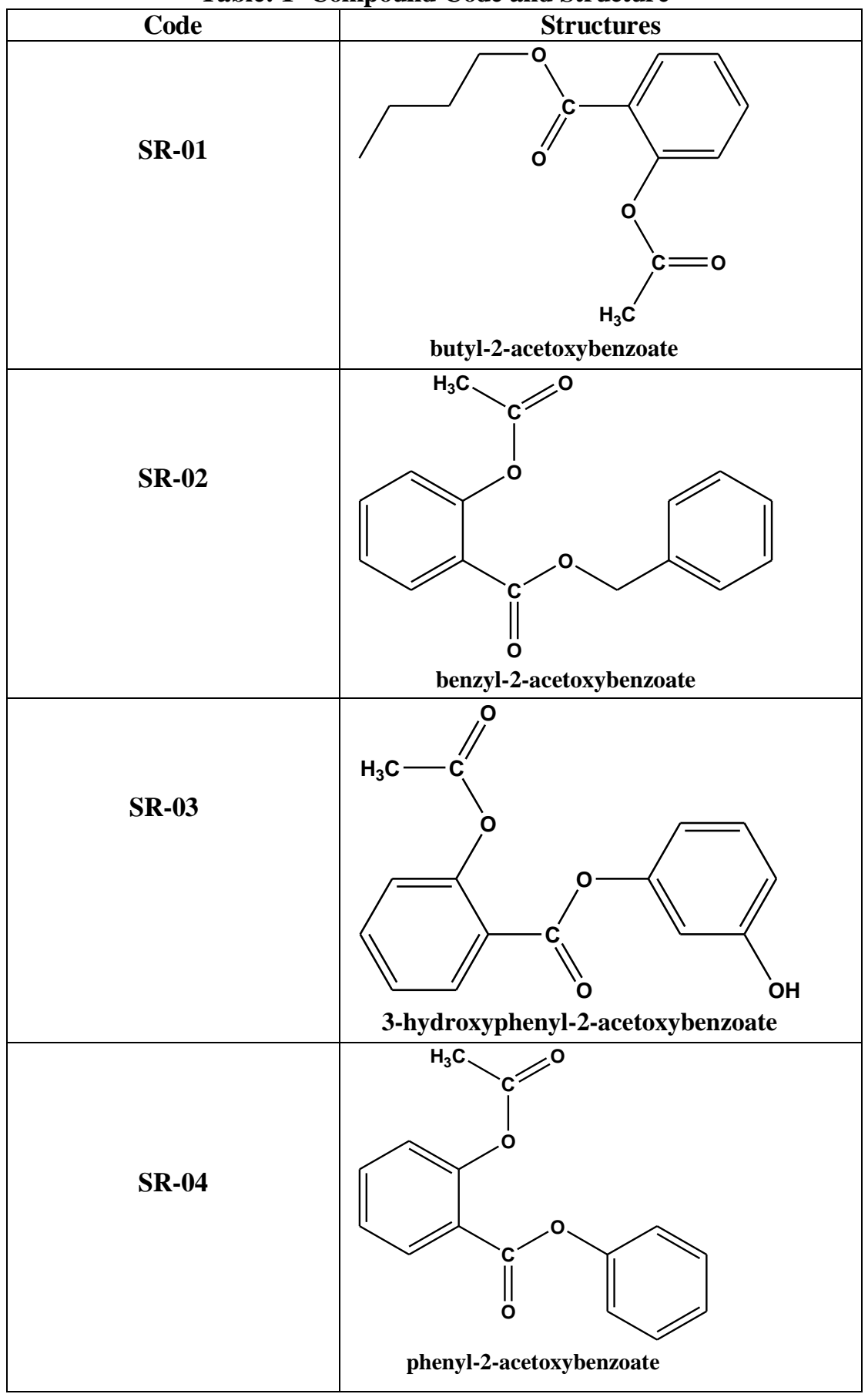




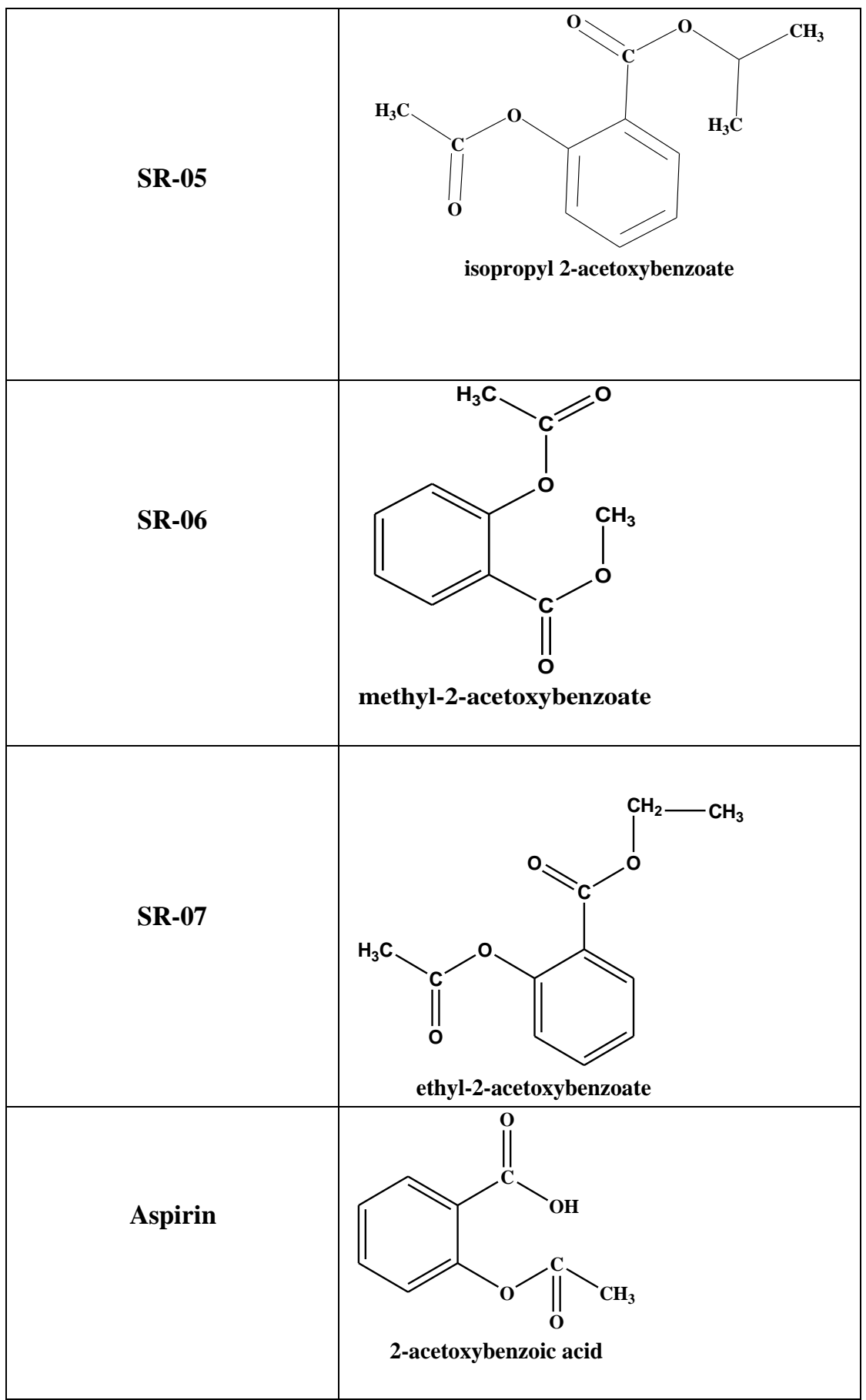

Table 2:- Dock Score \& Hydrogen Bond Energy of Aspirin \& Aspirin Derivatives

\begin{tabular}{|c|c|c|}
\hline Compound code & $\begin{array}{c}\text { Molecular } \\
\text { dock score }\end{array}$ & $\begin{array}{c}\text { Hydrogen bond } \\
\text { energy } \\
\text { (Kilo Joules) }\end{array}$ \\
\hline Aspirin & -66.64 & -6.24 \\
\hline SR-01 & -68.62 & 0 \\
\hline SR-02 & -89.44 & -2.50 \\
\hline SR-03 & $\mathbf{- 9 9 . 0 7}$ & $-\mathbf{7 . 1 7}$ \\
\hline SR-04 & -84.27 & -2.12 \\
\hline SR-05 & -73.24 & -1.24 \\
\hline SR-06 & -71.05 & -5.33 \\
\hline SR-07 & -71.43 & -1.25 \\
\hline
\end{tabular}




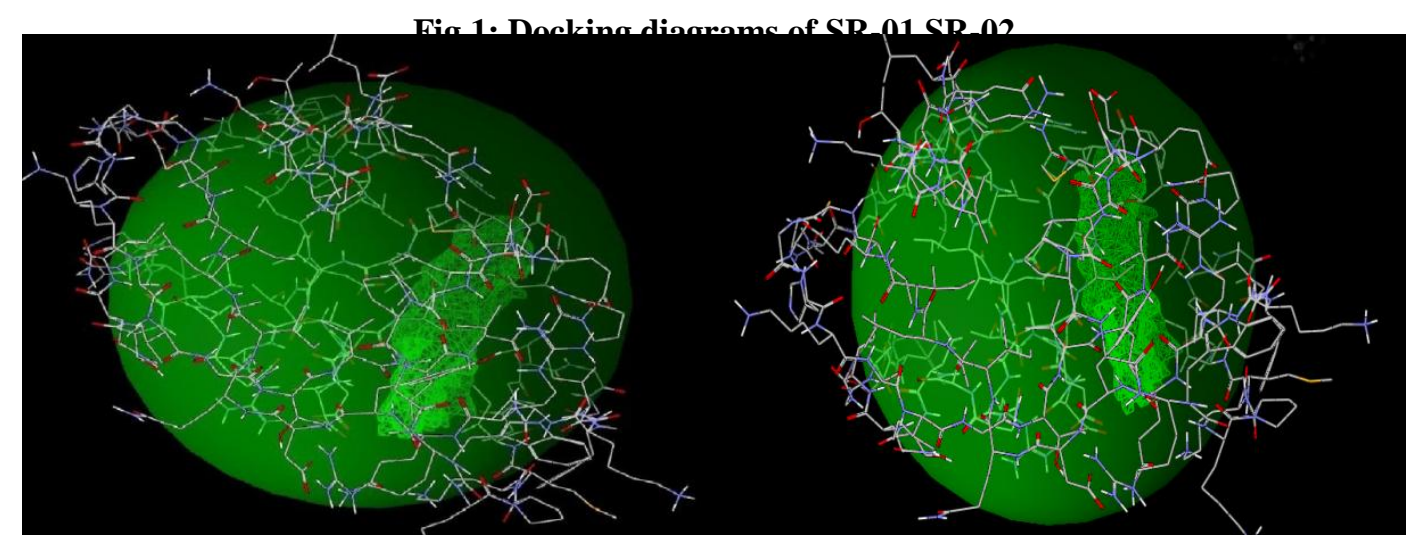

SR-01

SR-02

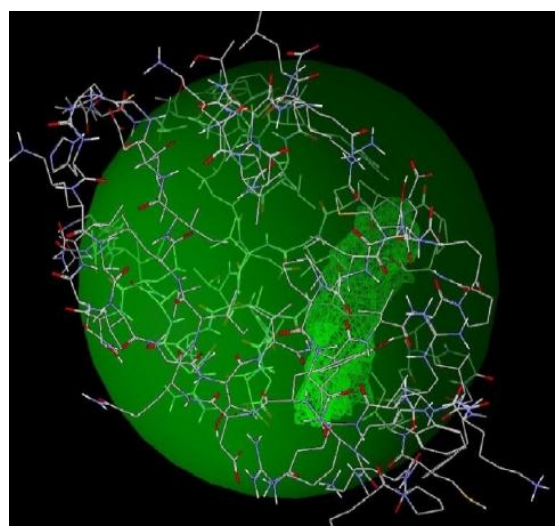

SR-03(Best docked structure with highest dock score and hydrogen bond energy)

Fig 2: Docking diagram of SR-03

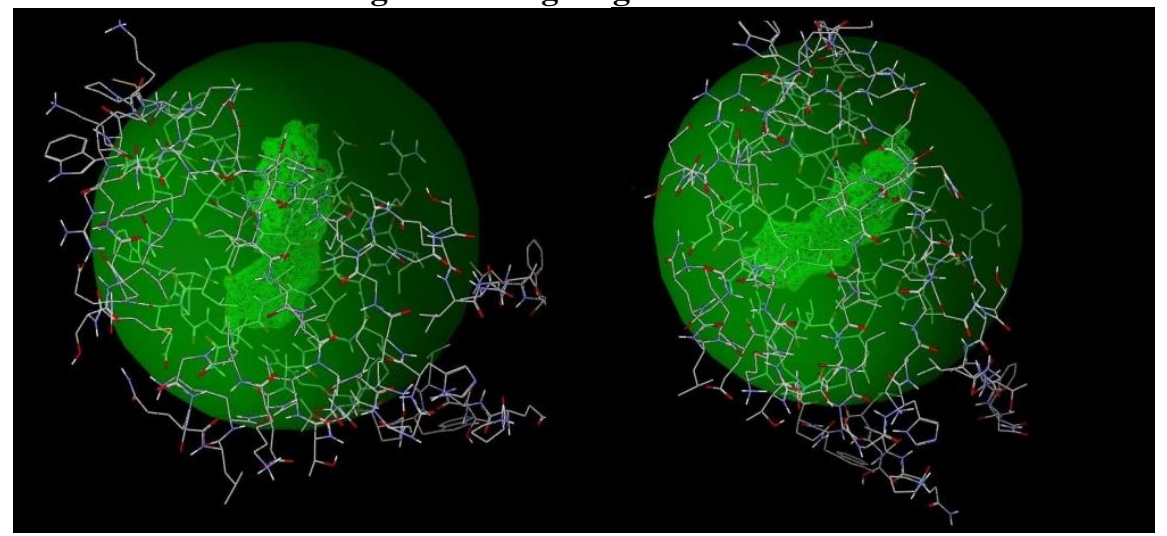

SR-04

SR-05

Fig 3: Docking diagrams of SR-04,SR-05 


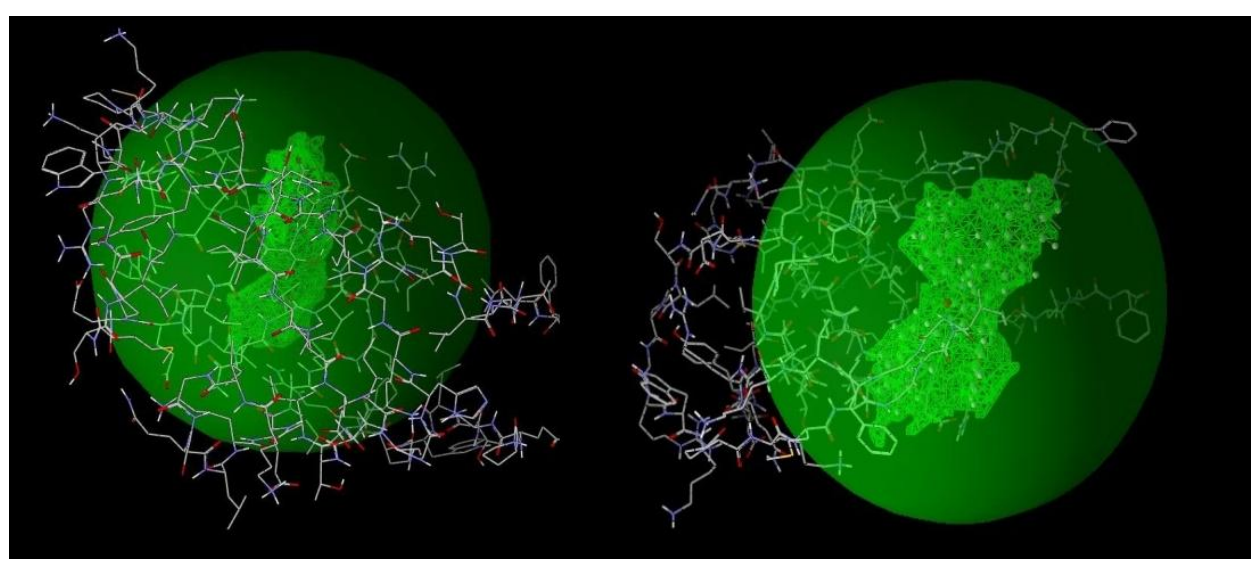

SR-06

SR-07

Fig 4: Docking diagrams of SR-06,SR-07

\section{Results And Discussion}

The docking studies were performed on HVR protein (HIV-1 protease) and from the studies it was found that the compound SR-03[Resorcinol derivative (dock score -99.07 and hydrogen bond energy $-7.17 \mathrm{KJ}$ )] was found to have the greatest affinity to HVR protein (HIV-1 protease) followed by SR-02[Benzyl derivative (dock score -89.44 and hydrogen bond energy $-2.50 \mathrm{KJ}$ )] and SR-04[Phenol derivative (dock score -84.27 and hydrogen bond energy $-2.12 \mathrm{KJ}$ )].The compounds SR-05[Isopropyl derivative (dock score -73.24 and hydrogen bond energy -1.24 KJ)], SR-06[Methyl derivative( dock score -71.05 and hydrogen bond energy -5.33 KJ)], SR07[ethyl derivative( dock score -71.43 and hydrogen bond energy $-1.25 \mathrm{KJ}$ )] showed moderate affinity towards HVR protein while Aspirin (dock score -66.64 and hydrogen bond energy -6.24 KJ), SR-01[Butyl derivative( dock score -68.62 and hydrogen bond energy $0 \mathrm{KJ}$ ) showed least affinity towards HVR protein.

It can be concluded theoretically that electron withdrawing groups attached to aryl substituent in carboxylic group of aspirin are likely to enhance affinity of aspirin to HVR protein while the electron releasing groups decreases the affinity of aspirin to HVR protein respectively. In brief it can be concluded that electron withdrawing groups attached to aryl substituent in carboxylic group of aspirin is responsible for pharmacological effect while electron donating groups are not so.

www.link.springer.com.

\section{References}

\section{CumentProteinandPeptideScience, 2007(8),312-328.}

www.utdallasedu.

www.scibdicom

www.msiumnedu.

AnIntroduction to MolecularDocking by Paul Sans chagin, 22.11.10.

www.pdb.org. 